\title{
On the Minimum Phase Property of Prediction-Error Polynomials
}

\author{
P. P. Vaidyanathan, Fellow, IEEE, J. Tuqan, Student Member, IEEE, and A. Kirac, Student Member, IEEE
}

\begin{abstract}
We provide a simple proof of the minimum phase property of the optimum linear prediction polynomial. The proof follows directly from the fact that the minimized prediction error has to satisfy the orthogonality principle. Additional insights provided by this proof are also discussed.
\end{abstract}

\section{INTRODUCTION}

$\mathbf{I}$ $\mathrm{N}$ THE theory of linear prediction, it is well known [1] that the zeros of the optimal prediction-error polynomial $A_{N}(z)$ are inside the unit circle. This is called the minimum-phase property of the polynomial $A_{N}(z)$. The most commonly known proof of this result goes as follows. First, the coefficients $k_{m}$ that arise in Levinson's recursion (parcor-coefficients) are shown to satisfy $\left|k_{m}\right|<1$ (the implicit assumption being that the process is not fully predictable; otherwise, one of the lattice coefficients has unit magnitude). Next, these coefficients are shown to be the multipliers in the infinite impulse response (IIR) lattice structure associated with the inverse linear predictive coefficient (LPC) filter. Using $\left|k_{m}\right|<$ 1 , it is finally shown that the lattice structure has all its poles inside the unit circle. Since these poles are also the zeros of $A_{N}(z)$, this completes the proof. A lucid treatment of this topic can be found in [2].

Simpler proofs of the minimum phase property have been advanced by a number of authors [3]-[5]. The elegant proofs advanced by Lang and McClellan [4] and later by Pakula and Kay [5] start from the simple observation that $A_{N}(z)$ can be factored into the form $C_{N-1}(z)\left(1-q z^{-1}\right)$. In this letter, we will still use this as a valuable starting point and obtain a new proof, which we believe to be the simplest. It follows directly from orthogonality principle.

To formulate the problem, consider Fig. 1 where $A_{N}(z)$ is a finite impulse response (FIR) filter of order $N$, given by $A_{N}(z)=1+\sum_{k=1}^{N} a_{k} z^{-k}$. This is the $N$ th order optimal prediction-error polynomial for the zero-mean wide sense stationary (WSS) process $x(n)$. If $x(n)$ is the input to $A_{N}(z)$, then the output is the prediction error $e_{N}(n)$. Optimality of the predictor means that $E\left|e_{N}(n)\right|^{2}$ has been minimized. According to the orthogonality principle, the optimal predictor

Manuscript received September 12, 1996. This work was supported in part by the ONR under Grant N00014-93-1-0231, by Tektronix, Inc., and by Rockwell International. The associate editor coordinating the review of this manuscript and approving it for publication was Prof. V. J. Mathews.

The authors are with the Department of Electrical Engineering, California Institute of Technology, Pasadena, CA 91125 USA (e-mail: ppvnath@sys.caltech.edu).

Publisher Item Identifier S 1070-9908(97)03580-3.

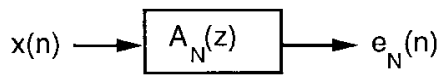

Fig. 1. Prediction-error polynomial viewed as an FIR filter.

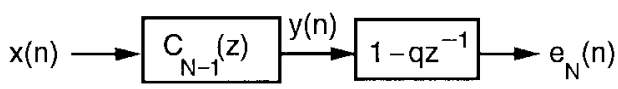

Fig. 2. Prediction-error filter with a zero factored out.

is such that the error $e_{N}(n)$ is orthogonal to the past $N$ samples

$$
E\left[e_{N}(n) x^{*}(n-k)\right]=0, \quad 1 \leq k \leq N .
$$

\section{A Simplified Proof of the Minimum-Phase Property}

Let $q$ be any zero of $A_{N}(z)$. Then we can redraw Fig. 1 as in Fig. 2 where $C_{N-1}(z)=1+\sum_{k=1}^{N-1} c_{k} z^{-k}$. Denoting the output of $C_{N-1}(z)$ as $y(n)$, we see that the sample $y(n-1)$ is the linear combination $y(n-1)=x(n-1)+c_{1} x(n-2)+$ $\cdots+c_{N-1} x(n-N)$. In view of the orthogonality property (1), it follows that

$$
E\left[e_{N}(n) y^{*}(n-1)\right]=0 .
$$

That is, the prediction error sample $e_{N}(n)$ is orthogonal to the sample $y(n-1)$. Next, since

$$
e_{N}(n)=y(n)-q y(n-1)
$$

we have $E\left[e_{N}(n) y^{*}(n-1)\right]=E\left[y(n) y^{*}(n-1)\right]-q E[y(n-$ 1) $y^{*}(n-1)$ ]. Letting $R_{y y}(k)$ denote the autocorrelation of the WSS process $y(n)$ and using the orthogonality condition (2), it then follows that

$$
0=R_{y y}(1)-q R_{y y}(0) .
$$

From (3)

$$
\begin{aligned}
E\left[\left|e_{N}(n)\right|^{2}\right] & =E\left[e_{N}(n)(y(n)-q y(n-1))^{*}\right] \\
& =E\left[e_{N}(n) y^{*}(n)\right] \quad(\text { from orthogonality (2)) } \\
& =R_{y y}(0)-q R_{y y}^{*}(1) \quad(\text { from (3)) } \\
& =R_{y y}(0)\left(1-|q|^{2}\right) \quad(\text { from (4)). }
\end{aligned}
$$

It is at this point that we need to assume that the process $x(n)$ is not fully predictable, which implies that $E\left[\left|e_{N}(n)\right|^{2}\right]>0$. 
Then, the above right-hand side is positive. In particular, we have $R_{y y}(0)>0$ and $|q|<1$. Thus, for the case where $x(n)$ is not fully predictable, we have shown that $q$ is strictly inside the unit circle. (For the fully predictable case it is known that there exists a predictor polynomial with all zeros on the unit circle, such that the prediction error is zero [6].)

\section{CONCLUDING REMARKS}

For the case where $x(n)$ is not fully predictable, it also follows from (4) that $q=R_{y y}(1) / R_{y y}(0)$, since $R_{y y}(0) \neq 0$. The derivation of this expression can in fact be simplified by the following observation: If $A_{N}(z)$ is optimal for the process $x(n)$, then it should be true that $\left(1-q z^{-1}\right)$ is optimal for the process $y(n)$. If this were not the case, one would be able to replace $\left(1-q z^{-1}\right)$ with another polynomial $\left(1-\rho z^{-1}\right)$ whose output (in response to the input $y(n)$ ) has a smaller mean square value. But this would imply that the $N$ th order polynomial $C_{N-1}(z)\left(1-\rho z^{-1}\right)$ produces a smaller prediction error for the original process $x(n)$ than does $A_{N}(z)$, thus contradicting the optimality of $A_{N}(z)$. In conclusion, every factor $\left(1-q z^{-1}\right)$ of the polynomial $A_{N}(z)$ is the optimal prediction error filter for the random process filtered through the remaining factors. Since the best first-order prediction coefficient is the correlation coeffcient of its input process, this gives a second justification of $q=R_{y y}(1) / R_{y y}(0)$.

\section{ACKNOWLEDGMENT}

The authors thank J. Makhoul for his enthusiasm for the above improved proofs. The main ideas of this letter evolved out of a beginning year graduate course taught by the first author at California Institute of Technology in the spring of 1996.

\section{REFERENCES}

[1] J. Makhoul, "Linear prediction: A tutorial review," Proc. IEEE, vol. 63, pp. 561-580 Apr. 1975.

[2] J. G. Proakis and D. G. Manolakis, Digital Signal Processing. Englewood Cliffs, NJ: Prentice-Hall, 1996.

[3] S. Treitel and T. J. Ulrych, "A new proof of the minimum-phase property of the unit prediction error operator," IEEE Trans. Acoust., Speech, Signal Processing, vol. ASSP-27, pp. 99-100, Feb. 1979.

[4] S. W. Lang and J. H. McClellan, "A simple proof of stability for all-pole linear prediction models," Proc. IEEE, vol. 67, pp. 860-861, May 1979.

[5] L. Pakula and S. Kay, "Simple proofs of the minimum-phase property of the prediction error filter," IEEE Trans. Acoust., Speech, Signal Processing, vol. ASSP-31, p. 501, Apr. 1983.

[6] A. Papoulis, "Predictable processes and Wold's decomposition: A review," IEEE Trans. Acoust., Speech, Signal Processing, vol. ASSP-33, pp. 933-938, Aug. 1985. 